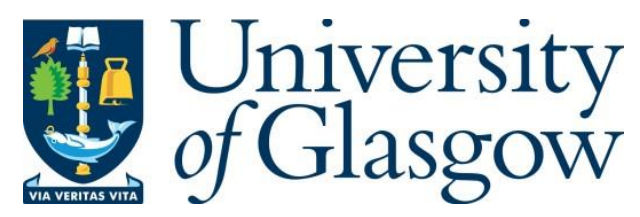

Kawai, Y., Uchiyama, K. and McInnes, C. R. (2018) Design Principle of Non-Switching Integral Sliding Mode Controller and Applications to Aerospace Vehicles. In: 2018 AIAA Guidance, Navigation, and Control Conference, Kissimmee, FL, USA, 08-12 Jan 2018, ISBN 9781624105265.

There may be differences between this version and the published version. You are advised to consult the publisher's version if you wish to cite from it.

http://eprints.gla.ac.uk/155544/

Deposited on: 17 January 2018

Enlighten - Research publications by members of the University of Glasgow http://eprints.gla.ac.uk 


\title{
Design Principle of Non-Switching Integral Sliding Mode Controller and Applications to Aerospace Vehicles
}

\author{
Yuki Kawai ${ }^{1}$ and Kenji Uchiyama ${ }^{2}$ \\ Nihon University, Funabashi, Chiba, 274-8501, Japan \\ Colin R. McInnes ${ }^{3}$ \\ University of Glasgow, Glasgow, Scotland, G12 8QQ, United Kingdom
}

This paper proposes a new type of sliding mode controller termed non-switching integral sliding mode controller. It shows a certain robustness of the control system against matched uncertainty with non-switching input. Furthermore, it allows the control system to be analyzed from the aspects of the system norms, small gain theorem, and structured singular value. The validities of the proposed controller are shown by performing the numerical simulations for three different applications: a spacecraft in a displaced non-Keplerian orbit, a soft-landing problem, and a quadcopter carrying a manipulator. The potential of the proposed sliding mode control methodology is demonstrated numerically for versatility in the applications.

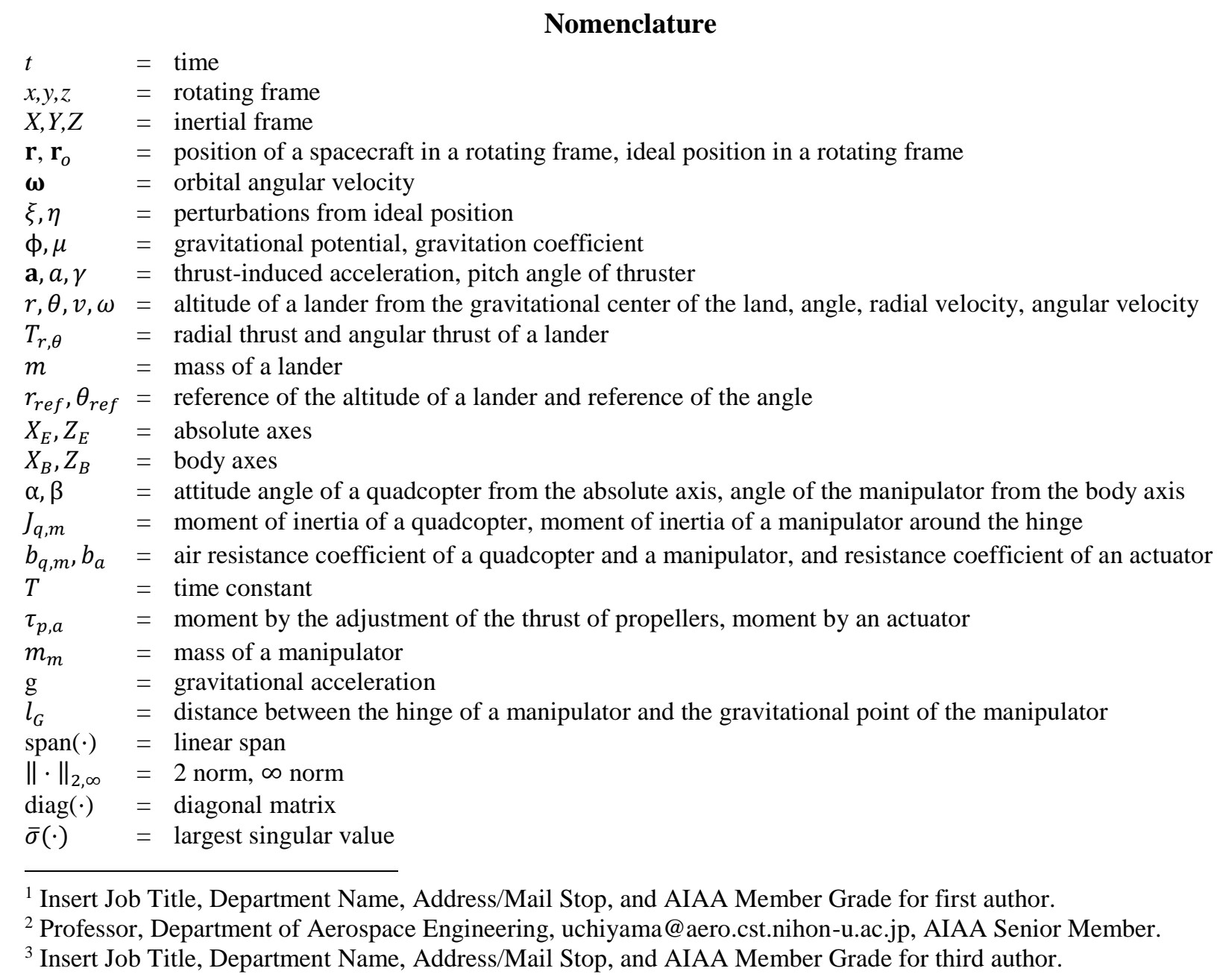




\section{Introduction}

Sliding mode, $\mathrm{H}_{\infty}$, and $\mu$ controls have been used as robust controls. Sliding mode control guarantees asymptotic

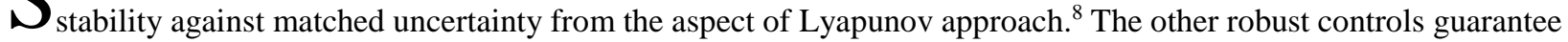
robust stability (RS) and robust performance (RP) from the aspects of the system norms, small gain theorem, and structured singular value. ${ }^{12}$ General sliding mode control potentially has the disadvantage of the chattering caused by a switching input. Therefore, chattering-free methods have been proposed. ${ }^{1-9}$ The common methods to overcome the chattering are suppressing the magnitude of input near the sliding surface and in high frequency range. However, these smoothed sliding controllers cannot guarantee asymptotic stability from Lyapunov approach against the magnitudebounded uncertainty that satisfies the matching condition for general sliding mode control, or the sliding modes experience lose of generality, then, the controllers force the system into undesirable modes. That kind of problem caused by smoothing the switching input is unavoidable when using Lyapunov approach. Therefore, a new type of sliding mode controller that shows the robustness of the control system by a non-switching input is required.

The proposed controller in this paper termed non-switching integral sliding mode controller (NS-ISMC) is an integral sliding mode controller ${ }^{1}$ with a non-switching input. Conventional smoothed sliding mode control methods cannot show any certainty of robustness against the magnitude-bounded uncertainty that satisfies the matching condition for general sliding mode control but the NS-ISMC shows the robustness of the control system against magnitude-bouded uncertainty and even unbounded uncertainty. In addition, the NS-ISMC allows RS and RP to be analyzed from the aspects of the system norms, small gain theorem, and structured singular value if the controlled system is analyzable. Thus, the NS-ISMC has the possibility to guarantee RS and RP in the same way as $\mathrm{H}_{\infty}$ and $\mu$ controls. Conventional sliding mode control have been discussed as robust control in different fields from them because it cannot be analyzed for RS and RP from the same aspect. Thus, it can be said that the NS-ISMC is a type of sliding mode control to be discussed in the same field as $\mathrm{H}_{\infty}$ and $\mu$ controls for the first time.

The validities of the NS-ISMC are shown by using three different applications: a spacecraft in a displaced nonKeplerian orbit, a soft-landing problem, and a quadcopter carrying a manipulator. They show the design of the NSISMC for linearized input, an affine system, and guaranteeing robust stability and performance. These numerical simulations were performed in MATLAB.

\section{Non-switching integral sliding mode control theory}

Non-switching integral sliding mode controller (NS-ISMC), a new type of sliding mode controller, will now be introduced by describing the system and matching conditions, the form of the controller, and its robustness and design.

\section{A. System description and matching conditions}

The dynamics of the controlled system is described as below.

$$
\dot{\mathbf{x}}=\mathbf{A}(\mathbf{x})+\mathbf{B}(\mathbf{x}, \mathbf{u})+\mathbf{d}
$$

$\mathbf{x}, \mathbf{u}, \mathbf{d}$ are the state, input, and disturbance vector, respectively. An integral sliding mode is designed as below.

$$
\dot{\mathbf{x}}_{\mathbf{s}}=\mathbf{A}_{\mathbf{s}}(\mathbf{x})+\mathbf{B}_{\mathbf{s}}(\mathbf{x}, \mathbf{r})
$$

$\mathbf{r}$ is the reference vector. The following two matching conditions have to be satisfied to design the NS-ISMC.

$$
\mathbf{H}(\mathbf{x}, \mathbf{d}, \mathbf{r}) \in \operatorname{span}\left\{\mathbf{B}_{\mathbf{v}}(\mathbf{x})\right\}, \boldsymbol{\sigma}_{\mathbf{r}}(\mathbf{x}) \in\left\{\boldsymbol{\Sigma}_{\mathbf{r}}(\mathbf{x}) \mid \frac{\partial \boldsymbol{\Sigma}_{\mathbf{r}}(\mathbf{x})}{\partial \mathbf{x}} \mathbf{B}_{\mathbf{v}}(\mathbf{x})=\text { diagonal fixed matrix }\right\}
$$

$\mathbf{H}$ is the uncertain vector given as below and $\mathbf{B}_{\mathbf{v}}$ the virtual input matrix.

$$
\mathbf{H}=\mathbf{A}+\mathbf{B}+\mathbf{d}-\mathbf{A}_{\mathbf{s}}-\mathbf{B}_{\mathbf{s}}-\mathbf{B}_{\mathbf{v}}\left(\mathbf{u}-\mathbf{u}_{\mathbf{n}}\right)
$$

$\mathbf{u}_{\mathbf{n}}$ is the nominal input vector added in the input vector $\mathbf{u}$. The nominal input vector is not essential but it has the possibility to reduce the uncertain vector beforehand. The first matching condition given in Eq.(3) is for uncertainty to satisfy the possibility of cancellation. The second matching condition is for robust vector to guarantee the decoupled transfer function explained later. 


\section{B. Form of controller}

The NS-ISMC can be designed with reaching vector $\boldsymbol{\sigma}$.

$$
\mathbf{u}=\mathbf{u}_{\mathbf{n}}-\boldsymbol{\sigma}, \boldsymbol{\sigma}=\sigma_{\mathbf{r}}+\sigma_{\mathbf{i}}, \sigma_{\mathbf{i}}=-\int \frac{\partial \sigma_{\mathbf{r}}}{\partial \mathbf{x}}\left(A_{\mathbf{s}}(\mathbf{x})+\mathbf{B}_{\mathbf{s}}(\mathbf{x}, \mathbf{r})\right) \mathrm{dt}, \sigma_{\mathbf{i}}(0)=-\sigma_{\mathbf{r}}(\mathbf{x}(0))
$$

where $\boldsymbol{\sigma}_{\mathbf{r}}$ is a robust vector which must satisfy its matching condition given in Eq.(3) and $\boldsymbol{\sigma}_{\mathbf{i}}$ an integral vector which handles the integral sliding mode. The dynamics of the reaching vector can be derived from Eqs.(1), (4), and (5) as follows:

$$
\dot{\boldsymbol{\sigma}}=\frac{\partial \sigma_{\mathrm{r}}}{\partial \mathbf{x}} \dot{\mathbf{x}}+\dot{\boldsymbol{\sigma}}_{\mathbf{i}}=\frac{\partial \sigma_{\mathbf{r}}}{\partial \mathbf{x}}\left(\mathbf{H}-\mathbf{B}_{\mathbf{v}} \boldsymbol{\sigma}\right)
$$

The reaching vector can be expressed by the channel-reduced uncertain vector $\mathbf{h}: \mathbf{H}=\mathbf{B}_{\mathbf{v}} \mathbf{h}$ as Laplace form as below.

$$
\boldsymbol{\sigma}(\mathrm{s})=\left(\mathrm{s} \mathbf{I}+\frac{\partial \sigma_{\mathbf{r}}}{\partial \mathbf{x}} \mathbf{B}_{\mathbf{v}}\right)^{-1} \frac{\partial \sigma_{\mathbf{r}}}{\partial \mathbf{x}} \mathbf{B}_{\mathbf{v}} \mathbf{h}(\mathrm{s})
$$

\section{Robustness and design}

An error vector is used to discuss the robustness of the control system. The error vector $\mathbf{e}_{\mathbf{h}}$ : $\dot{\mathbf{x}}-\dot{\mathbf{x}}_{\mathbf{s}}=\mathbf{B}_{\mathbf{v}} \mathbf{e}_{\mathbf{h}}$ can be derived from Eqs.(1), (2), (4), (5), and (7).

$$
\mathbf{e}_{\mathbf{h}}(\mathrm{s})=\mathrm{s}\left(\mathrm{s} \mathbf{I}+\frac{\partial \sigma_{\mathbf{r}}}{\partial \mathbf{x}} \mathbf{B}_{\mathbf{v}}\right)^{-1} \mathbf{h}(\mathrm{s})
$$

It is possible to design a complexed shaped filter from each channel-reduced uncertainty to each error by designing $\boldsymbol{\sigma}_{\mathbf{r}}$ depending on frequency, but frequency-independent design is focused on in this paper. In that case, Eq.(8) shows that the NS-ISMC can suppress the channel-reduced uncertain vector to get through from the virtual input matrix into the ideal system $\dot{\mathbf{x}}=\mathbf{A}_{\mathbf{s}}(\mathbf{x})+\mathbf{B}_{\mathbf{s}}(\mathbf{x}, \mathbf{r})$ in the low frequency range because the decoupled transfer function from each channel-reduced uncertainty to each error is a high pass filter. Incidentally, constant uncertainty is eliminated. Needless to say, the robustness against the uncertain vector depends on the robust vector $\boldsymbol{\sigma}_{\mathbf{r}}$. The input vector can be expressed with the nominal input vector and the uncertain vector as below.

$$
\mathbf{u}(s)=\mathbf{u}_{\mathbf{n}}-\left(\mathrm{s} \mathbf{I}+\frac{\partial \sigma_{\mathbf{r}}}{\partial \mathbf{x}} \mathbf{B}_{\mathbf{v}}\right)^{-1} \frac{\partial \sigma_{\mathbf{r}}}{\partial \mathbf{x}} \mathbf{B}_{\mathbf{v}} \mathbf{h}(\mathrm{s})
$$

It is obvious that the input vector would have high magnitude in the high frequency range if the cut-off frequency of Eq.(8) has been set too high. However, the reduction of the uncertain vector in high frequency range may not be required because its effects may be small enough for the ideal system. Therefore, the robust vector should be designed by considering which frequency range the uncertain vector should be suppressed in while avoiding rough input from Eqs.(8) and (9). The design of the NS-ISMC basically requires only the consideration of frequency range rather than that of magnitude of the input because it shows its robustness against unbounded uncertainty unlike all other sliding mode controller.

To choose the virtual input matrix $\mathbf{B}_{\mathbf{v}}$ decides which part of the input is considered as a part of the uncertain vector for the reaching vector while ignoring the nominal input vector like $\mathbf{B}(\boldsymbol{\sigma})-\mathbf{B}_{\mathbf{v}} \boldsymbol{\sigma}$. If the virtual input matrix is too different from the actual input matrix, it is impossible for the reaching vector to cover the uncertain vector. If it is designed as $\mathbf{B}_{\mathbf{v}}=\partial \mathbf{B} / \partial \mathbf{u}$, no part of the input is considered as an uncertainty. It might be designed as $\mathbf{B}_{\mathbf{v}}=$ $(\partial \mathbf{B} / \partial \mathbf{u})_{\mathbf{u}=\mathbf{u}_{\mathbf{n}}}$ with stationary input $\mathbf{u}_{\mathbf{n}}$ so that the approximation error is considered as an uncertainty. It may be better to choose the decoupled virtual input matrix even though $\partial \mathbf{B} / \partial \mathbf{u}$ is a coupled form because each reaching vector can handle the uncertainty of each channel separately.

Eq.(8) does not actually guarantee RS or RP by itself. But most importantly, because the controller would be a continuous linear form, the NS-ISMC allows RS and RP to be analyzed by system norms, small-gain theorem, and structured singular value unlike all other sliding mode controller if the controlled system is analyzable. Therefore, it can be said that the NS-ISMC made it possible to handle a type of sliding mode control in the same filed as $\mathrm{H}_{\infty}$ and $\mu$ controls for the first time. 


\section{Applications}

In this paper, the NS-ISMC is applied in three scenarios: a spacecraft in a displaced non-Keplerian orbit, a softlanding problem, and a quadcopter carrying a manipulator. The design of NS-ISMC for linearized input, an affine system, and guaranteeing robust stability and performance is demonstrated by them. Each numerical simulation result is performed in MATLAB.

\section{A spacecraft in a displaced non-Keplerian orbit (Linearized input)}

If the input of the system is a nonlinear form as $\mathbf{B}(\mathbf{u}) \neq \frac{\partial \mathbf{B}}{\partial \mathbf{u}} \mathbf{u}$, a virtual input matrix cannot be chosen as an anctual input matrix. In that case, it has to be chosen by linearizing the input. A spacecraft in a displaced non-Keplerian orbit was chosen as an example of this system.

The stability of the displaced non-Keplerian orbits of Fig. 1 was discussed. ${ }^{10}$ A spacecraft on unstable displaced orbits should be controlled. Here, the NS-ISMC is used to stabilize it.

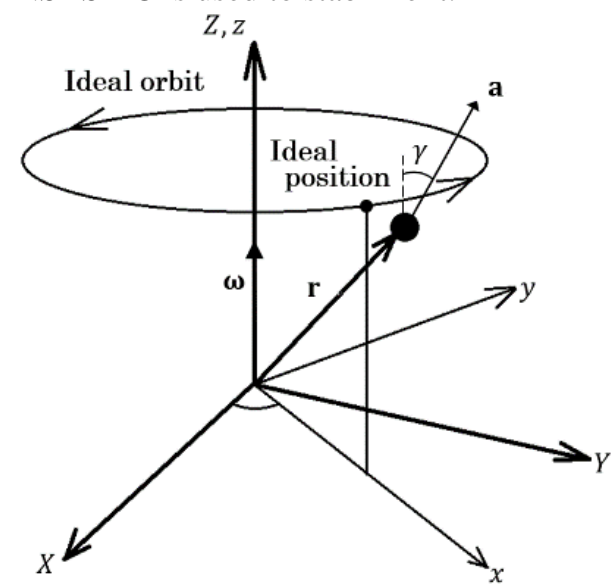

Figure 1. A spacecraft in a displaced non-Keplerian orbit with thrust-induced acceleration

The conditions for a displaced non-Keplerian orbit are now investigated by considering the dynamics of a spacecraft at position $\mathbf{r}$ in a rotating frame $(x, y, z)$. This frame rotates with the constant angular velocity of ideal orbit $\boldsymbol{\omega}=\omega_{o} \hat{\mathbf{z}}$ relative to an inertial frame $(X, Y, Z)$. The equation of motion of the spacecraft on the rotating frame is given with thrust-induced acceleration $\mathbf{a}$ as below.

$$
\ddot{\mathbf{r}}+2 \boldsymbol{\omega} \times \dot{\mathbf{r}}+\boldsymbol{\omega} \times(\boldsymbol{\omega} \times \mathbf{r})=\mathbf{a}-\boldsymbol{\nabla} \boldsymbol{\phi}, \mathbf{a}=\left[\begin{array}{lll}
a \sin \gamma & 0 & a \cos \gamma
\end{array}\right]^{T}, \phi=-\left(\mu /\|\mathbf{r}\|_{2}\right)
$$

The required stationary pitch angle $\gamma_{o}$ and thrust-induced acceleration $a_{o}$ needed to fix the spacecraft in the displaced orbit are given as $\ddot{\mathbf{r}}=\dot{\mathbf{r}}=\mathbf{0}$ at $\mathbf{r}=\mathbf{r}_{o}\left(x_{o}, 0, z_{o}\right)$.

$$
\tan \gamma_{o}=\frac{x_{o}}{z_{o}}\left(1-\frac{\omega_{o}^{2}\left\|\mathbf{r}_{o}\right\|_{2}^{3}}{\mu}\right), a_{o}=\sqrt{x_{o}{ }^{2}\left(\omega_{o}{ }^{2}-\frac{\mu}{\left.\left\|\mathbf{r}_{o}\right\|_{2}\right)^{2}}+z_{o}{ }^{2} \frac{\mu^{2}}{\left\|\mathbf{r}_{o}\right\|_{2}{ }^{6}}\right.}
$$

The dynamics of perturbations can be given by linearizing Eq.(10) around ideal position $\mathbf{r}_{o}$ and stationary input $\mathbf{a}_{o}{ }^{10}$

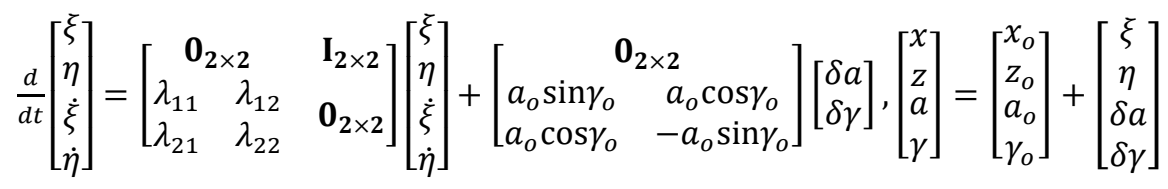

$\lambda_{i j}$ is the matrix elements derived from $\omega_{0}, \mathbf{r}_{o}$, and $\mu$. If a PD controller is designed against Eq.(12), the approximation error of the input is too big against big perturbations because it is obviously impossible to maintain linearity against trigonometric function at the condition. However, if the NS-ISMC is designed against the linearized input, the perturbational control input would be 0 at the initial condition even if the perturbations are big at that time, and the magnitude of the perturbational control input is not determined by just the position and velocity but the uncertain vector unlike PD-controllers. Therefore, the NS-ISMC has possibility to maintain the linearlity of the input. 
Here, the NS-ISMC will now be designed against a spacecraft in a displaced non-Keplerian orbit. The integral sliding mode, virtual input matrix, and uncertain vector are given by

$$
\dot{\mathbf{x}}_{\mathbf{s}}=\left[\begin{array}{cccc}
\mathbf{0}_{2 \times 2} & \multicolumn{2}{c}{\mathbf{I}_{2 \times 2}} \\
-A_{\xi p} & 0 & -A_{\xi d} & 0 \\
0 & -A_{\eta p} & 0 & -A_{\eta d}
\end{array}\right]\left[\begin{array}{l}
\xi \\
\eta \\
\dot{\xi} \\
\dot{\eta}
\end{array}\right], \mathbf{B}_{\mathbf{v}}=\left[\begin{array}{cc}
\mathbf{0}_{2 \times 2} \\
a_{o} \sin \gamma_{o} & a_{o} \cos \gamma_{o} \\
a_{o} \cos \gamma_{o} & -a_{o} \sin \gamma_{o}
\end{array}\right], \mathbf{H}=\frac{\mathrm{d}}{\mathrm{dt}}\left[\begin{array}{c}
\xi \\
\eta \\
\dot{\xi} \\
\dot{\eta}
\end{array}\right]-\dot{\mathbf{x}}_{\mathbf{s}}+\mathbf{B}_{\mathbf{v}} \boldsymbol{\sigma}
$$

The virtual input matrix was designed as a coupled form because the superiority of each input for each channel is obscure. The approximation error of the input is considered as uncertainty. The input vector, robust vector, and error vector are given by

$$
\left[\begin{array}{l}
a \\
\gamma
\end{array}\right]=\left[\begin{array}{l}
a_{o} \\
\gamma_{o}
\end{array}\right]-\boldsymbol{\sigma}, \boldsymbol{\sigma}_{\mathbf{r}}=\left[\begin{array}{cc}
K_{\xi} & 0 \\
0 & K_{\eta}
\end{array}\right]\left[\begin{array}{ll}
a_{o} \sin \gamma_{o} & a_{o} \cos \gamma_{o} \\
a_{o} \cos \gamma_{o} & -a_{o} \sin \gamma_{o}
\end{array}\right]^{-\mathbf{1}}\left[\begin{array}{c}
\dot{\xi} \\
\dot{\eta}
\end{array}\right], \mathbf{e}_{\mathbf{h}}(\mathrm{s})=\mathrm{s}\left(\mathrm{s} \mathbf{I}+\left[\begin{array}{cc}
K_{\xi} & 0 \\
0 & K_{\eta}
\end{array}\right]\right)^{-1} \mathbf{h}(\mathrm{s})
$$

where $K_{\xi}, K_{\eta}$ are the parameters of the controller. The stationary input was added as the nominal input vector.

The results of the numerical simulation are shown in Fig.5 and the used parameters are shown in Table 1. The unstable displaced orbits: Orbit 1 and 2 could be controlled by the NS-ISMC. The PD controllers based on pole placement, the poles of which are the same as the ones of the NS-ISMC, could not make it stable because $\delta \gamma$ is too large to maintain the linearity of trigonometric function. As representative, the responses of perturbations and inputs of the spacecraft controlled by the NS-ISMC in Orbit 1 are shown. The high robustness against nonlinearity of input can be seen.

\section{B. Soft-landing problem (Affine system)}

When the NS-ISMC is designed against an affine system without any part of the input being considered as an uncertainty, the robust vector has to be chosen so that it satisfies its matching condition with the virtual input matrix $\mathbf{B}_{\mathbf{v}}(\mathbf{x}) \neq \mathbf{B}_{\mathbf{v}}(\mathbf{0})$. A soft-landing problem shown in Fig.2 was chosen as an example of the system.

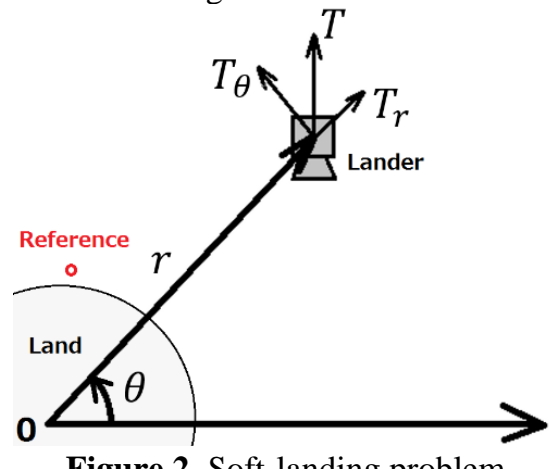

Figure 2. Soft-landing problem

The dynamics of the soft landing problem of Fig. 2 are shown as below as an affine system. ${ }^{11}$

$$
\dot{\mathbf{x}}=\mathbf{A}(\mathbf{x})+\mathbf{B}(\mathbf{x}) \mathbf{T}, \mathbf{x}=\left[\begin{array}{c}
r \\
\theta \\
v \\
\omega
\end{array}\right], \mathbf{T}=\left[\begin{array}{c}
T_{r} \\
T_{\theta}
\end{array}\right], \mathbf{A}(\mathbf{x})=\left[\begin{array}{c}
v \\
\omega \\
-\frac{\mu}{r^{2}}+r \omega^{2} \\
-\frac{2 v \omega}{r}
\end{array}\right], \mathbf{B}(\mathbf{x})=\left[\begin{array}{cc}
\mathbf{0}_{2 \times 2} \\
\frac{1}{m} & 0 \\
0 & \frac{1}{m r}
\end{array}\right]
$$

The integral sliding mode of the NS-ISMC is given as below.

$$
\dot{\mathbf{x}}_{\mathbf{s}}=\mathbf{A}_{\mathbf{s}} \mathbf{x}+\mathbf{B}_{\mathbf{s}} \mathbf{r}, \mathbf{r}=\left[\begin{array}{c}
r_{r e f} \\
\theta_{r e f}
\end{array}\right], \mathbf{A}_{\mathbf{s}}=\left[\begin{array}{cccc}
\mathbf{0}_{2 \times 2} & \mathbf{I}_{2 \times 2} \\
-K_{r} & -C_{r} & 0 & 0 \\
0 & 0 & -K_{\theta} & -C_{\theta}
\end{array}\right], \mathbf{B}_{\mathbf{s}}=\left[\begin{array}{cc}
\mathbf{0}_{2 \times 2} \\
K_{r} & 0 \\
0 & K_{\theta}
\end{array}\right]
$$

The virtual input matrix and robust vector are given as below. 


$$
\mathbf{B}_{\mathbf{v}}(\mathbf{x})=\left[\begin{array}{cc}
\mathbf{0}_{2 \times 2} \\
\frac{1}{m} & 0 \\
0 & \frac{1}{m r}
\end{array}\right], \boldsymbol{\sigma}_{\mathbf{r}}(\mathbf{x})=\left[\begin{array}{c}
K_{1} m v \\
K_{2} m r \omega
\end{array}\right]
$$

$K_{1}, K_{2}$ are the parameters of the robust vector. The virtual input matrix was designed so that it equals the actual input matrix. Thus, no part of the input is considered as an uncertainty. The robust vector was designed so as to satisfy its matching condition given in Eq.(3). The input vector, error vector, and uncertain vector are given as below.

$$
\mathbf{T}=-\boldsymbol{\sigma}, \mathbf{e}_{\mathbf{h}}(\mathrm{s})=\left[\begin{array}{cc}
\frac{\mathrm{s}}{\mathrm{s}+K_{1}} & 0 \\
0 & \frac{\mathrm{s}}{\mathrm{s}+K_{2}}
\end{array}\right] \mathbf{h}(\mathrm{s}), \mathbf{H}=\mathbf{A} \mathbf{x}-\mathbf{A}_{\mathbf{s}} \mathbf{x}-\mathbf{B}_{\mathbf{s}} \mathbf{r}
$$

The input vector $\mathbf{T}$ was designed without nominal input vector.

The results of the numerical simulation are shown in Fig.6 and the used parameters are shown in Table 2. The NSISMC could achieve its mission for each reference without steady state error by smooth input. It can be seen that the radial input eliminates gravitational term, constant uncertainty at the end positions. The NS-ISMC has certain robustness against constant uncertainty like this because of Eq.(8).

\section{Quadcopter carrying a manipulator (NS-ISMC guaranteeing robust stability and performance)}

The NS-ISMC has the possibility to guarantee RS and RP if the controlled system is analyzable. Here, a quadcopter carrying a manipulator is considered as the application.

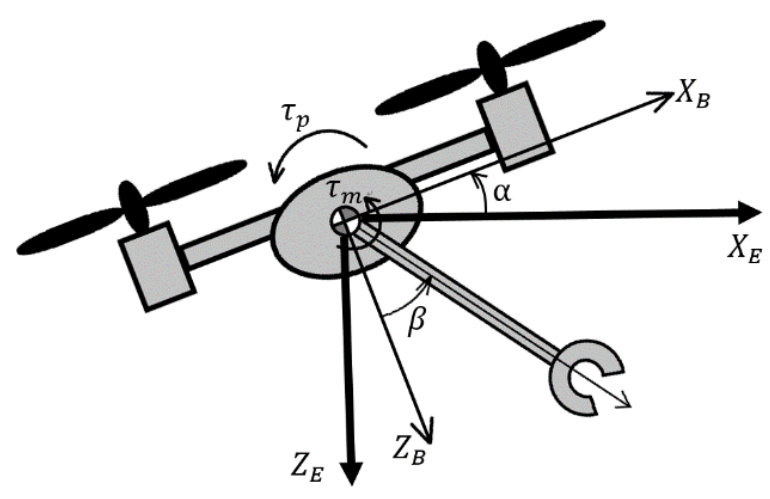

Figure 3. A quadcopter carrying a manipulator

The dynamics of the quadcopter carrying a manipulator in Fig. 3 can be described by considering action and reaction with the delay of the propeller input as below.

$$
\begin{gathered}
\dot{\mathbf{x}}=\mathbf{A x}+\mathbf{B} \mathbf{u}^{\prime}+\mathbf{B}_{\mathrm{d}} \mathrm{d}, \mathbf{x}=\left[\begin{array}{llll}
\alpha & \beta & \dot{\alpha} & \dot{\beta}
\end{array}\right]^{T}, \mathbf{u}^{\prime}(\mathrm{s})=\left[\begin{array}{cc}
\frac{1}{T s+1} & 0 \\
0 & 1
\end{array}\right] \mathbf{u}(\mathrm{s}), \mathbf{u}=\left[\begin{array}{ll}
\tau_{p} & \tau_{a}
\end{array}\right]^{T}, \mathrm{~d}=\sin (\alpha+\beta) \\
\mathbf{A}=\left[\begin{array}{ccc}
\mathbf{0}_{2 \times 2} & -\frac{b_{q}}{J_{q}} & \frac{\mathbf{b}_{a}}{J_{q}} \\
\mathbf{0}_{2 \times 2} & \frac{b_{q}}{J_{q}}-\frac{b_{m}}{J_{m}} & -\frac{J_{q}+J_{m}}{J_{q} J_{m}} b_{a}-\frac{b_{m}}{J_{m}}
\end{array}\right], \mathbf{B}=\left[\begin{array}{cc}
\mathbf{0}_{2 \times 2} \\
\frac{1}{J_{q}} & -\frac{1}{J_{q}} \\
-\frac{1}{J_{q}} & \frac{J_{m}+J_{q}}{J_{m} J_{q}}
\end{array}\right], \mathbf{B}_{\mathrm{d}}=\left[\begin{array}{c}
\mathbf{0}_{3, \mathbf{1}} \\
-\frac{\mathrm{m}_{\mathrm{mgl}}}{\mathrm{J}_{\mathrm{m}}}
\end{array}\right]
\end{gathered}
$$

$J_{q}, J_{m}, b_{q}, b_{m}, b_{a}, T$ are the parameters of the system having parametric uncertainty. The NS-ISMC was designed to achieve RS and RP against parametric uncertainty and exogenous signals.

The integral sliding mode and virtual input matrix are given as below. 


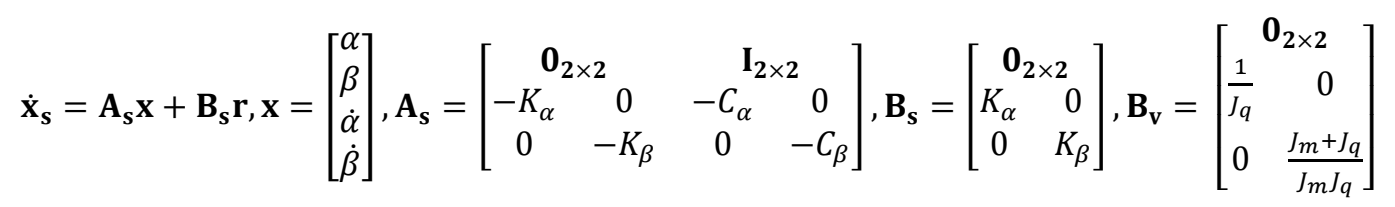

$K_{\alpha}, K_{\beta}, C_{\alpha}, C_{\beta}$ are the parameters of the integral sliding mode. The virtual input matrix has been designed as a decoupled form to let each reaching vector handle the uncertainty of each channel separately. Furthermore, it ignores the delay of the thrust of the propeller. Thus, the uncertain vector includes the effects of the first order delay of the input by the propellers. It causes uncertainty in the high frequency range. The input vector designed with nominal input vector $\mathbf{u}_{\mathbf{n}}$, the uncertain vector $\mathbf{H}$, and the state vector in Laplace form are given as below.

$$
\mathbf{u}=\mathbf{u}_{\mathbf{n}}-\boldsymbol{\sigma}, \mathbf{H}=\left(\mathbf{A}-\mathbf{A}_{\mathbf{s}}\right) \mathbf{x}-\mathbf{B}_{\mathbf{s}} \mathbf{r}+\mathbf{B u} \mathbf{u}^{\prime}+\mathbf{d}+\mathbf{B}_{\mathbf{v}} \boldsymbol{\sigma}, \mathbf{x}(\mathrm{s})=\left(\mathrm{sI}-\mathbf{A}_{\mathbf{s}}\right)^{-1} \mathbf{B}_{\mathbf{s}} \mathbf{r}(\mathrm{s})+\left(\mathrm{sI}-\mathbf{A}_{\mathbf{s}}\right)^{-1} \mathbf{B}_{\mathbf{v}} \mathbf{e}_{\mathbf{h}}(\mathrm{s})
$$

The nominal input vector $\mathbf{u}_{\mathbf{n}}=\mathbf{K}_{\mathrm{x}} \mathbf{x}+\mathbf{K}_{\mathbf{r}} \mathbf{r}$ is designed by pole placement against the nominal model ignoring the first order delay of the propeller input. The poles were placed at the same positions as the integral sliding mode given in Eq.(20). It was added in the input vector to enhance total robustness by reducing the uncertain vector beforehand.

The RS and RP of the system in parameter variations was analyzed by $\mu$-analysis. It is the method to evaluate RS and RP by using the structured singular value $\mu_{\Delta}(\mathbf{M})$ defined as

$$
\mu_{\Delta}^{-1}(\mathbf{M}):=\min _{\Delta \in \Delta_{\mathbf{s}}}\{\bar{\sigma}(\Delta): \operatorname{det}(\mathbf{I}-\mathbf{M} \Delta)=0\}
$$

where the nominal plant, structured uncertain block, and structured uncertain block sets are denoted respectively by $\mathbf{M}, \Delta, \Delta_{\mathbf{s}}$. If there is no $\Delta \in \Delta_{\boldsymbol{s}}$ such that $\operatorname{det}(\mathbf{I}-\mathbf{M} \Delta)=0$, then $\mu_{\Delta}(\mathbf{M}):=0{ }^{12}$

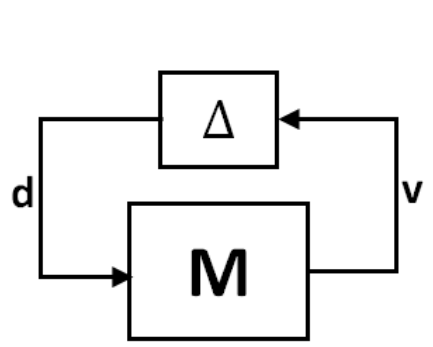

(a) $\mathrm{M}-\Delta$ configuration for $\mathbf{R S}$

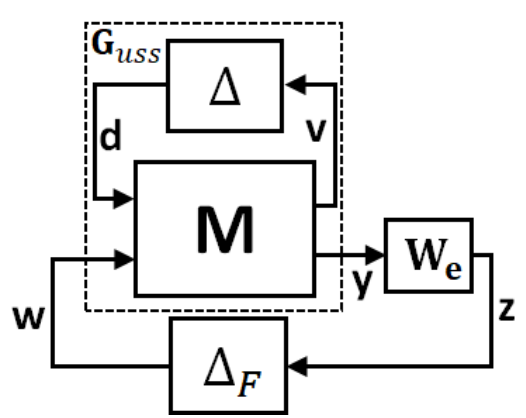

(b) $\mathbf{M}-\Delta_{P}$ configuration for $\mathbf{R P}$

\section{Figure 4. Standard configuration for RS and RP analysis}

It is assumed that the uncertain block has been normalized in the rest of this paper, i.e. $\|\Delta\|_{\infty} \leq 1$. In Fig.4, $\mathbf{w}, \mathbf{z}, \mathbf{v}$, $\mathbf{d}, \mathbf{W}$ are, respectively, the exogenous input, error output, input of the dynamic uncertainties, output of the dynamic uncertainties, and weighting matrix. RS is guaranteed when $\mu_{\Delta}(\mathbf{M})<1$ is fulfilled against $\mathbf{M}-\Delta$ configuration for RS in Fig.4. RP is guaranteed when $\mu_{\Delta_{P}}(\mathbf{M})<1$ is fulfilled against $\mathbf{M}-\Delta_{P}$ configuration for RP in Fig.4. $\mathbf{M}-\Delta_{P}$ configuration for RP is extended from $\mathbf{M}-\Delta$ configuration for RS to handle the exogenous input and error output with extended uncertain block $\Delta_{P}$ consisting of the structured uncertain block $\Delta$ and a fictitious complex unstructured uncertain block $\Delta_{F}$ satisfying $\left\|\Delta_{F}\right\|_{\infty} \leq 1$ so as to be $\Delta_{P}=\operatorname{diag}\left(\Delta, \Delta_{F}\right)$. $\mathbf{G}_{\text {uss }}$ has the form with respect to the integral sliding mode, delay of input, and control input as $\left[\begin{array}{llll}\dot{\mathbf{x}}_{\mathbf{s}} & \dot{\mathbf{x}} & \tau_{p}{ }^{\prime} & \mathbf{y}\end{array}\right]^{T}=\mathbf{G}_{u s s}\left[\begin{array}{llll}\mathbf{x}_{\mathbf{s}} & \mathbf{x} & \tau_{p}{ }^{\prime} & \mathbf{w}\end{array}\right]^{T}$.

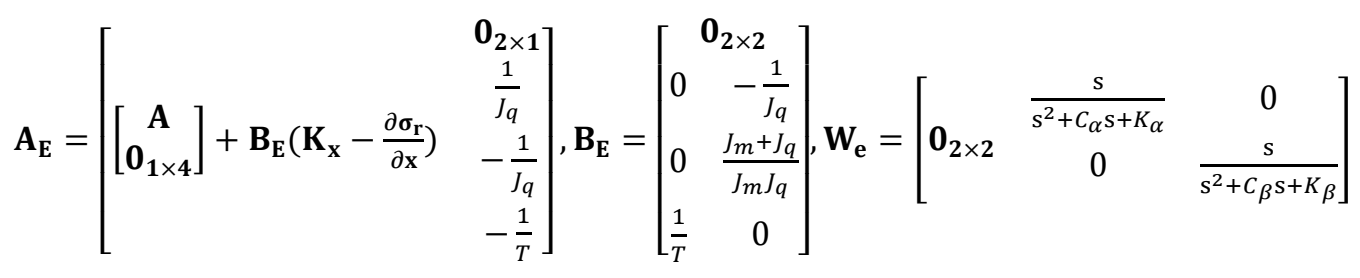




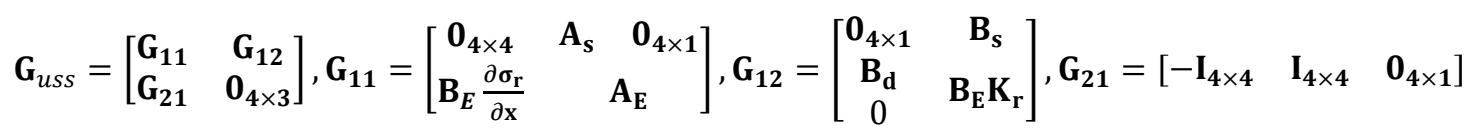

The error output, and exogenous input was chosen as $\left[\begin{array}{ll}\mathbf{I} & \mathbf{0}_{2 \times 2}\end{array}\right]\left(\mathbf{s} \mathbf{I}-\mathbf{A}_{\mathbf{s}}\right)^{-1} \mathbf{B}_{\mathbf{v}} \mathbf{e}_{\mathbf{h}},\left[\begin{array}{ll}\mathrm{d} & \mathbf{r}\end{array}\right]^{T}$ by considering Eq.(21).

It is difficult to compute a structured singular value itself. Therefore, the upper bound and lower bound of a structured singular value are usually computed. That kind of analysis of RS and RP for the system was done in MATLAB. The results of the $\mu$-analysis, used parameters, and robust vector are shown in Table 3 . The RS and RP are obviously achieved. The step responses from each reference and disturbance to each angle, and the results of the numerical simulation of Eq.(19) in the presence of parametric uncertainty are shown in Fig.7. It is shown that each state behaves as each integral sliding mode with smooth input. It was shown that the NS-ISMC has the possibility to guarantee certain RS and RP.

\section{Conclusion}

A non-switching integral sliding mode controller (NS-ISMC) has been proposed as a new type of sliding mode controller. It indicates a certain robustness of the control system against matched uncertainty with a non-switching input. The NS-ISMC was applied to three different scenarios with the numerical simulation. The results showed that the proposed control method was valid. In the applications, the NS-ISMC guaranteeing robust stability and performance by $\mu$-analysis was designed. Thus, it is connoted that the NS-ISMC made it possible to discuss a type of sliding mode controller in the same field as $\mathrm{H}_{\infty}$ and $\mu$ controls for the first time.

\section{References}

${ }^{1}$ Utkin, V., Shi, J., "Integral Sliding Mode in Systems Operating under Uncertainty Conditions,” CDC, 13 Dec. 1996, pp. 45914596.

${ }^{2}$ Husain, A.R., Ahmad, M.N., Yatim, A.H.M,“Chattering-free Sliding Mode Control for an Active Magnetic Bearing System,” World Academy of Science, Engineering and Technology 39, 2008, pp. 385-391.

${ }^{3} \mathrm{Ma}$, D., Lin, H.,Li, B., "Chattering-Free Sliding-Mode Control for electromechanical Actuator with Backlash Nonlinear," JECE, Conference on Decision and Control, 13 Feb. 2017.

${ }^{4}$ Feng, Y., Han, F., Yu, X., “Chattering free full order sliding mode control,” Automatica, Apr. 2014, Vol. 50, pp. 1310-1314.

${ }^{5}$ Shaer, B., Kenne, J.P., Kaddissi, C., Fallaha, C., "A chattering-free fuzzy hybrid slidingmode control of an electrohydraulic active suspension," TIMC, 20 Jun. 2016.

${ }^{6} \mathrm{Gao}$, W., James, C.H., "Variable Structure Control of Nonlinear Systems: A New Approach,” IEEE, Transactions on industrial electronics, Vol. 40, No. 1, Feb. 1993, pp. 45-55.

${ }^{7}$ Utkin, V., Lee, H., "Chattering suppression methods in sliding mode control systems," Annual Reviews in Control 31, 2007, pp. $179-188$.

${ }^{8}$ Utkin, V., Guldner, J., Shi, J.,"Sliding Mode Control in Electromechanical Systems, Second Edition,” 1 May. 2009

${ }^{9}$ Kawai, Y, Uchiyama, K., "Modified Integral Sliding Mode Controller with Saturation Function,” ICMAE, 18-20 July. 2016, pp. 358-362.

${ }^{10}$ McInnes, C.R.,"Dynamics, Stability, and Control of Displaced Non-Keplerian Orbits” Journal of Guidance, Control, And Dynamics, Vol. 21, No. 5, Sep. 1998,799-805.

${ }^{11}$ Xinglong, L., Guangren, D., "Nonlinear Optimal Control for the Soft Landing of Lunar Lander," ISSCAA, 19-21 Jan. 2006, pp. 1382-1387.

${ }^{12}$ Gu, D.W., Petkov, P.H., Konstantinov, M.M., "Robust Control Design with MATLAB Second Edition,” 2013.

Table 1. Parameters A

$\begin{array}{cc}\mu\left[\mathrm{km}^{3} / \mathrm{s}^{2}\right] & 398600 \\ \omega_{o}[\mathrm{rad} / \mathrm{s}] & 7.3 \times 10^{-5} \\ A_{\xi p} & 1 \times 10^{-7} \\ A_{\xi d} & 0.001 \\ A_{\eta p} & 1 \times 10^{-7} \\ A_{\eta d} & 0.001 \\ K_{\xi} & 0.1 \\ K_{\eta} & 0.01 \\ (0), \eta(0)[\mathrm{km}] & 1000,1000 \\ \text { bit } 1 x_{o}, z_{o}[\mathrm{~km}] & 7000,2000 \\ \text { bit } 2 x_{o}, z_{o}[\mathrm{~km}] & 2000,7000\end{array}$

Table 2. Parameters B

$\begin{array}{cc}\mu\left[\mathrm{km}^{3} / \mathrm{s}^{2}\right] & 4903 \\ \mathrm{~m}[\mathrm{~kg}] & 600 \\ K_{1}, K_{2} & 3,1 \\ K_{r} & 5 \times 10^{-5} \\ C_{r} & 0.01 \\ K_{\theta} & 5 \times 10^{-5} \\ C_{\theta} & 0.01 \\ r(0), v(0) & 1.753 \times 10^{6}, 0 \\ \theta(0), \omega(0) & 0,9.64 \times 10^{-4} \\ r_{r e f}[\mathrm{~m}] & 1.738 \times 10^{6} \\ \theta_{\text {ref }}[\mathrm{deg}] & -30,30,60,90,120\end{array}$

Table 3. Parameters $\mathbf{C}$

\begin{tabular}{cc}
\hline$J_{q}, J_{m}\left[\mathrm{kgm}^{2}\right]$ & $0.002,0.001$ \\
& $\pm 30 \%$ \\
$b_{q}, b_{m}, b_{a}$ & $5,3,8\left(\times 10^{-3}\right)$ \\
& $\pm 50 \%$ \\
$T$ & $0.05 \pm 50 \%$ \\
$m_{m} \mathrm{gl} l_{G}[\mathrm{Nm}]$ & 0.05 \\
$\boldsymbol{\sigma}_{\mathbf{r}}$ & {$[0.15 \dot{\alpha} \quad 0.2 \dot{\beta}]^{T}$} \\
$K_{\alpha}, C_{\alpha}$ & 20,8 \\
$K_{\beta}, C_{\beta}$ & 10,5 \\
$\mu_{\Delta}^{-1}(\mathbf{M})(\mathrm{L}, \mathrm{H})$ & $1.6225,2.4376$ \\
$\mu_{\Delta_{P}}^{-1}(\mathbf{M})(\mathrm{L}, \mathrm{H})$ & $1.3804,1.9162$ \\
\hline
\end{tabular}




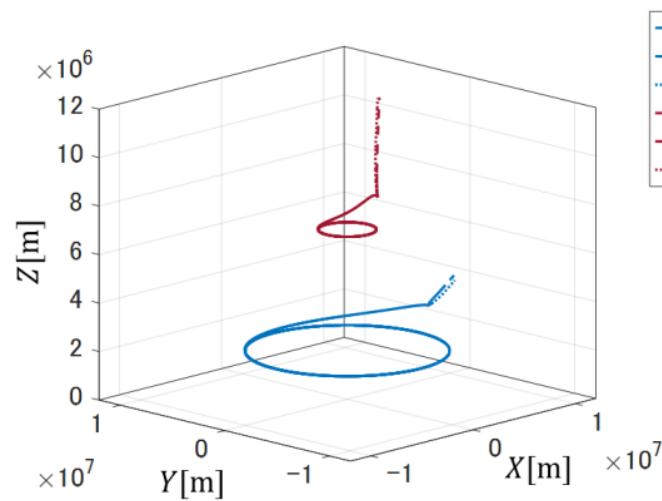

(a) Orbits of spacecraft

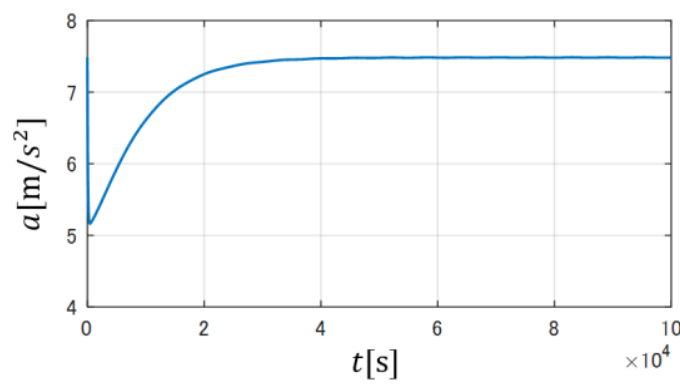

(c)Thrust reduced acceleration (Orbit 1)

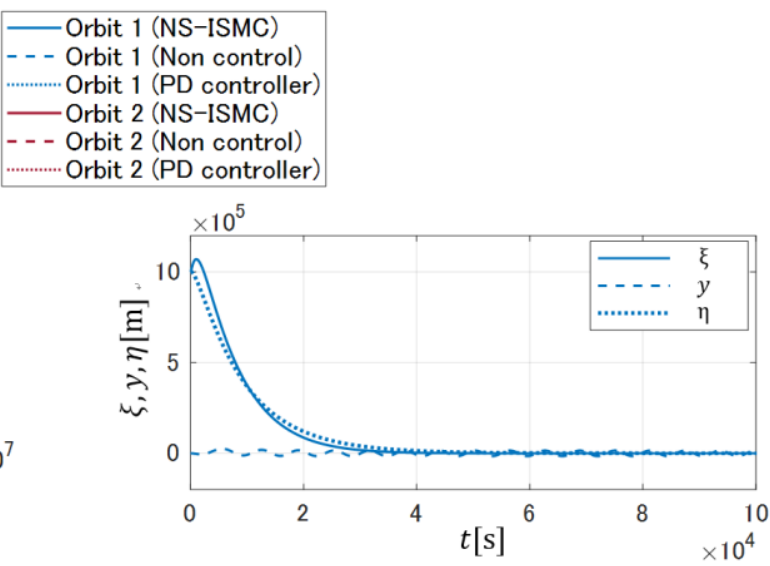

(b) Responses of perturbations (Orbit 1)

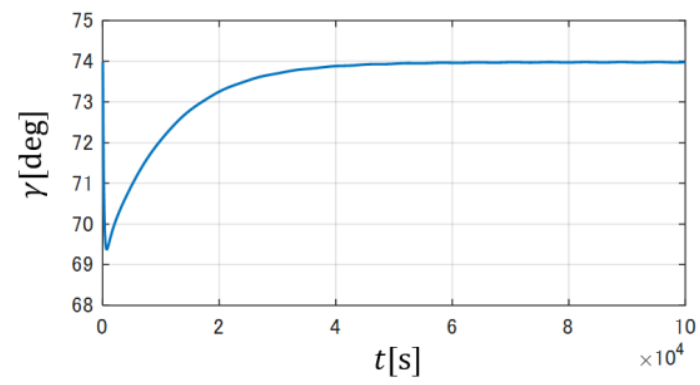

(d) Pitch Angle of thruster (Orbit 1)

Figure 5. Numerical simulation results of application A

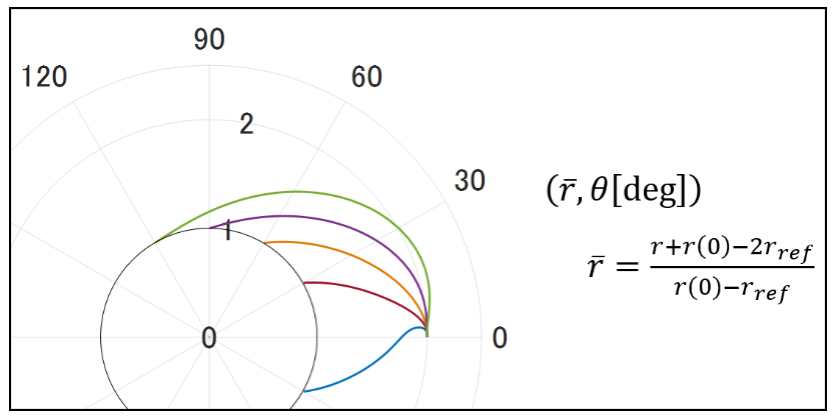

(a) Tracks of the lander

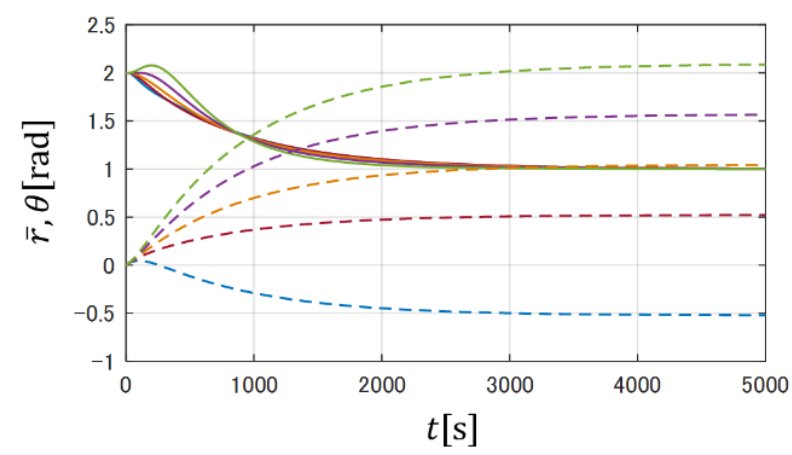

(b) Altitude and angle of lander ( $\bar{r}$ : solid line, $\theta$ : dashed line)

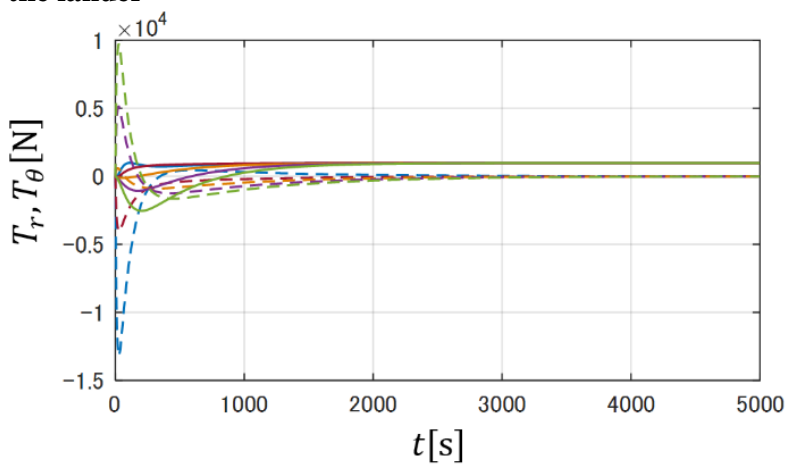

(c) Input of lander $\left(T_{r}\right.$ : solid line, $T_{\theta}$ : dashed line $)$

Figure 6. Numerical simulation results of application B 

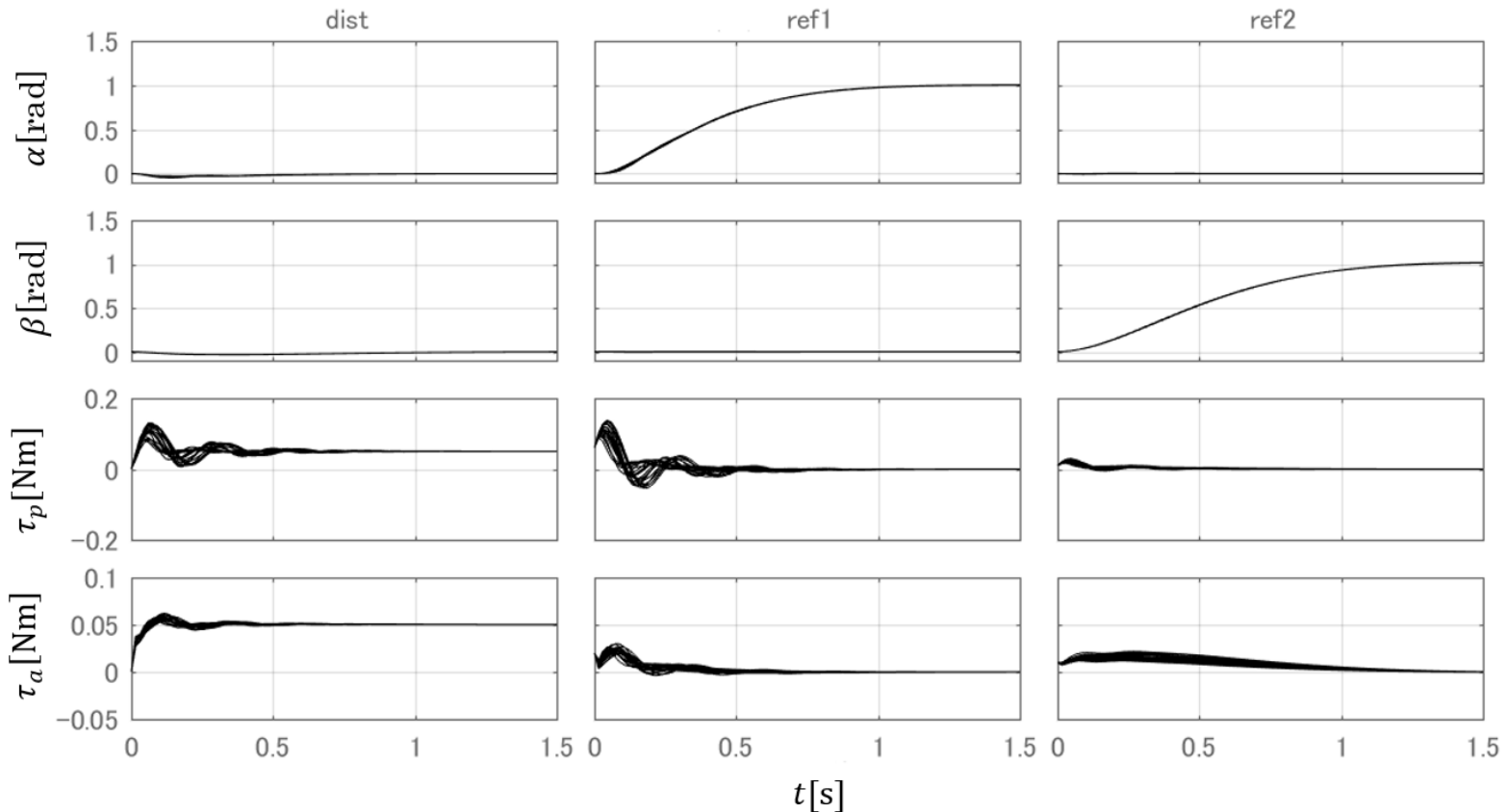

(a) Step responses from each reference or disturbance to each angle in parametric uncertainty
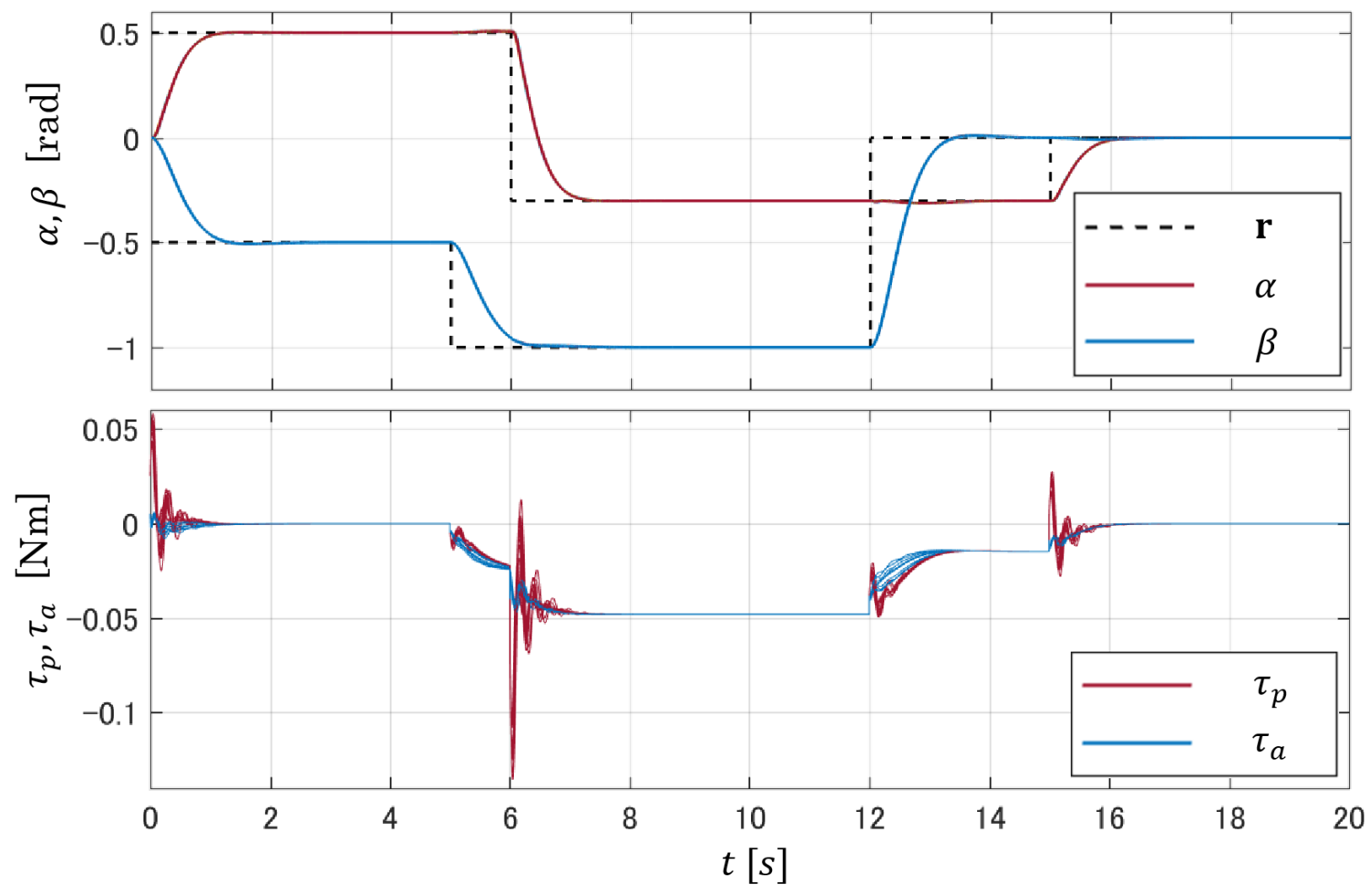

(b) Results of numerical simulation in parametric uncertainty

Figure 7. Numerical simulation results of application $\mathrm{C}$ 